Artigo Original

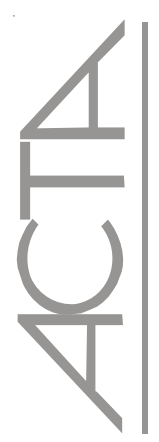

\title{
Paciente cirúrgico ambulatorial: calatonia e ansiedade*
}

\author{
The effect of calatonia on anxiety level of surgical outpatients
}

Paciente quirúrgico ambulatorio: calatonia y ansiedad

\section{Vitor Nosow ${ }^{1}$, Aparecida de Cássia Giani Peniche ${ }^{2}$}

\section{RESUMO}

Objetivo: consistiu em averiguar a eficácia do relaxamento promovido pela técnica de calatonia ao paciente no período pré-operatório. Métodos: a amostra constitui-se de 45 indivíduos alfabetizados, com idade superior a 17 anos, classificados como American Society of Anasthesiology 1 e divididos em 2 grupos: experimental (30) e controle (15). Foram entregues o Termo de Consentimento Livre e Esclarecido e a escala auto-aplicativa IDATE-estado; mensuraram-se os parâmetros vitais antes e após a técnica. Aplicou-se a técnica entre 1:30h e $30 \mathrm{~min}$ antes da cirurgia, com a duração de 10 minutos. Mediram-se e analisaram-se a freqüência de pulso, temperatura, freqüência respiratória e pressão arterial. Resultados: apenas a freqüência de pulso demonstrou redução estatisticamente significante $(\mathrm{p}=0,015)$. Conclusão: a técnica demonstrou eficácia, porém acredita-se que o curto tempo de aplicação, a imprecisão entre o questionário utilizado e a sua correspondência orgânica ou baixo nível de ansiedade dos grupos restringiram o relaxamento esperado no período pré-operatório.

Descritores: Cuidados pré-operatórios/psicologia; Assistência perioperatória; Ansiedade/terapia; Técnicas de relaxamento; Terapias complementares/métodos; Relações enfermeiro-paciente

\begin{abstract}
Objective: To determine the effectiveness of calatonia on anxiety level of surgical outpatients during the preoperative period. Methdos: the sample consists of 45 literate individuals at age of 17 years or over who were classified as American Society of Anasthesiology 1 and assigned to an experimental $(\mathrm{N}=30)$ or to a control group $(\mathrm{N}=15)$. Each subject signed an informed consent prior to being enrolled in the study. Pretest and posttest self-report measure of anxiety and vital signs were taken from subjects. Calatonia was delivered between 30 to 90 minutes before the surgery. Each section of calatonia had duration of 10 minutes. Results: only pulse rate had significant reduction $(p=0,015)$. Conclusion: the effect of calatonia on anxiety level might be effective. The short time of the delivery of the intervention, the incongruence between the score of the anxiety scale and systemic responses, and baseline low level of anxiety in both groups made it difficult to determine the effectiveness of the intervention on the anxiety level of surgical outpatients during the preoperative period.

Keywords: Preoperative care/psychology; Perioperative care; Anxiety/therapy; Relaxation techniques; Complemetary therapies/methods;
\end{abstract} Nurse-patient relations

\section{RESUMEN}

Objetivo: consistió en averiguar la eficacia del relajamiento promovido por la técnica de calatonia al paciente en el período preoperatorio. Métods: la muestra estuvo constituida por 45 individuos alfabetizados, con edad superior a los 17 años, clasificados como American Society of Anasthesiology 1 y divididos en 2 grupos: experimental (30) y control (15). Fueron entregados el consentimiento informado y la escala auto-aplicativa IDATE-estado; se midieron los signos vitales antes y después de la técnica. Se aplicó la técnica entre 1:30h y 30 minutos antes de la cirugía, con una duración de 10 minutos. Se midieron y analizaron la frecuencia del pulso, temperatura, frecuencia respiratoria y presión arterial. Resultados: apenas la frecuencia del pulso demostró reducción estadísticamente significativa $(\mathrm{p}=0,015)$. Conclusión: la técnica demostró eficacia, sin embargo se cree que el corto tiempo de aplicación, la imprecisión entre el cuestionario utilizado y su correspondencia orgánica o bajo nivel de ansiedad de los grupos restringieron el relajamiento esperado en el período preoperatorio.

Descriptores: Cuidados preoperatorios/psicología; Asistencia perioperatoria; Ansiedad/terapia; Técnicas de relajamiento; Terapias complementarias/métodos; Relaciones enfermero-paciente

* Trabalho realizado no Hospital Universitário da Universidade de São Paulo - USP - São Paulo, (SP), Brasil.

1 Acadêmico de Enfermagem e Bolsista FAPESP do Programa de Iniciação Científica da Universidade de São Paulo - USP - São Paulo (SP), Brasil.

${ }^{2}$ Professor Doutor do Departamento de Enfermagem Médico-Cirúrgica da Escola de Enfermagem da Universidade de São Paulo - USP - São Paulo (SP), Brasil. 


\section{INTRODUÇÃO}

A ansiedade é uma resposta fisiológica do organismo desencadeada por eventos estressantes, porém necessária para o indivíduo enfrentar as situações de risco. Em alguns momentos, como na realização de um procedimento anestésico cirúrgico, alterações no estado de ansiedade dos pacientes podem influenciar na recuperação ou até mesmo em todo o processo ocasionando alterações indesejáveis de parâmetros clínicos como, por exemplo, a elevação da pressão arterial. A redução dos níveis de ansiedade no período pré-operatório, em algumas situações, pode ser benéfica, tanto para o paciente crítico, como também para a equipe. Sendo assim, acredita-se que a aplicação de determinadas técnicas possa colaborar com esta redução da ansiedade. Das diversas linhas de terapias naturais ou complementares, ressurgiu a utilização das mãos como uma ferramenta eficiente. Várias são as técnicas utilizadas, abrangendo desde a pressão digitálica até o toque terapêutico, tendo nas massagens as mais conhecidas representantes dessa categoria.

O toque, com intensidade e localização préestabelecidas, com ou sem instrumentos, ocasiona reações diversas no organismo, podendo causar sedação, agitação, excitabilidade, relaxamento, prazer, dor, alegria, tristeza, extravasamento emocional (com posterior reequilíbrio emocional) entre outros efeitos, principalmente psicoemocionais $^{(1)}$.

Esse conhecimento, abandonado devido ao desenvolvimento de equipamentos mais sofisticados e fármacos potentes, vem sendo recuperado pelos profissionais da enfermagem que o vêem como importante recurso de interação com o paciente, tanto na criação de vínculos de empatia, quanto no auxílio à estabilização do equilíbrio físico-emocional ${ }^{(2)}$.

Sendo assim, considera-se o toque um instrumento possível de ser utilizado, não só nas tensões provocadas nos indivíduos pelos fatores estressantes do procedimento anestésico-cirúrgico, como também no seu estado de ansiedade decorrente deste processo.

A ansiedade, por ser uma resposta fisiológica a fatores estressantes, mobiliza todo o organismo para "luta-oufuga". Por estimulação nervosa originada no Sistema Nervoso Central (SNC), ocorre liberação seqüencial de adrenalina e hormônios corticóides pelo sistema nervoso simpático, preparando os órgãos, músculos e células para uma rápida solicitação com desempenho e resposta elevados. As mudanças ocorridas no corpo durante os estados de forte emoção são bastante semelhantes ${ }^{(3)}$. Modificações endócrinas, nervosas ou hormonais provocam rápidas respostas nos órgãos-alvo, o que possibilita o acompanhamento não invasivo do paciente em caso de medidas terapêuticas que objetivam a redução dos níveis de ansiedade.
Tendo em vista tais considerações, este estudo teve como finalidade a partir da utilização da calatonia, o relaxamento do paciente cirúrgico com provável diminuição do estado de ansiedade do paciente no período pré-operatório, colaborando com a intervenção anestésico-cirúrgica, na recuperação pós-operatória e nas possíveis complicações

Objetivos: Verificar o estado de ansiedade de pacientes no período pré-operatório; verificar os parâmetros clínicos associados à ansiedade no período pré-operatório antes da aplicação da técnica de relaxamento (calatonia) em pacientes; verificar os parâmetros clínicos associados à ansiedade no período pré-operatório após a aplicação da técnica de relaxamento (calatonia) em pacientes; relacionar os parâmetros clínicos obtidos pré e pós-aplicação da técnica de relaxamento (calatonia) em pacientes.

\section{MÉTODOS}

A pesquisa foi realizada no Hospital Universitário da Universidade de São Paulo, mais precisamente no Hospital Dia, abordando cirurgias ambulatoriais, após o consentimento do Comitê de Ética e Pesquisa da instituição. Os indivíduos foram esclarecidos quanto aos objetivos da pesquisa e dos procedimentos a serem realizados. A amostra foi composta de pacientes cirúrgicos submetidos à cirurgia ambulatorial, segundo os seguintes critérios de seleção: alfabetizados, com idade superior a 17 anos, submetidos à intervenção cirúrgica de pequeno e médio porte ${ }^{(4)}$ e classificados como ASA 1 ou ASA 2, de acordo com a American Society of Anesthesiology ${ }^{(5)}$. Devido à originalidade da técnica empregada e por não existir nenhum outro estudo na literatura com este enfoque, para este estudo foram selecionadas as cirurgias ambulatoriais devido ao baixo índice de disfunções sistêmicas dos pacientes, garantindo maior controle dos parâmetros utilizados nos questionários, e acarretando menores riscos ao paciente submetido à pesquisa. Constituiu-se de 45 indivíduos, sendo 30 do grupo experimental e 15 do grupo controle. Os 30 primeiros foram submetidos à aplicação da técnica nos pontos pré-determinados. Os 15 subseqüentes foram tocados em pontos diferentes, porém no mesmo sítio de interação. Foi utilizada como instrumento a escala referente ao estado de ansiedade, parte integrante do inventário validado e conhecido com Inventário de Ansiedade Traço-Estado (IDATE) ${ }^{(6)}$. Consta de 20 afirmações cujas intensidades são verificadas através de um escore de quatro pontos (1 a 4), tendo o seu total variando de 20 a 80 , onde os valores mais altos indicam um maior nível de ansiedade. Devido à oposição contida nas afirmativas, a pontuação segue a seguinte referência nos itens 3, 4, 6, 7, 9, 12,13,14, 17 e 18: valores crescentes 
(1 a 4) e nas afirmativas $1,2,5,8,10,11,15,16,19$ e 20: valores decrescentes (4 a 1 ).

Para avaliar os escores obtidos, utilizou-se a seguinte categorização $^{(7)}$ : de 20 a 40 pontos: baixa ansiedade; 41 a 60 pontos: média ansiedade; 61 a 80 pontos: alta ansiedade. Outro instrumento utilizado foi um formulário para o registro dos parâmetros clínicos antes e depois da aplicação da técnica, associados à atividade nervosa e endócrina desencadeada pela ansiedade ${ }^{(8)}$, de fácil mensuração e rápida detecção de alterações, constando de freqüência de pulso, pressão arterial, freqüência respiratória e temperatura. Os valores basais obtidos foram comparados aos valores correspondentes à normalidade e considerados por diversos autores ${ }^{(9-15)}$ como constitutivos de avaliação de pacientes cirúrgicos com o objetivo de observar variações que pudessem ocorrer entre as duas medidas realizadas em intervalos diferentes. A coleta de dados foi realizada em etapas distintas, isto é, uma entrevista inicial com o paciente após a internação, visando explicar o propósito da pesquisa, a técnica utilizada e sua aplicação, a participação voluntária e a garantia do anonimato. Cabe salientar que todas as etapas foram efetuadas pelo aluno, pois o mesmo realizou curso específico para a aplicação da técnica e dos questionários. Em caso afirmativo, solicitou-se a leitura e a confirmação da participação na pesquisa por meio do Termo de Consentimento Livre e Esclarecido. Após a entrevista, foi solicitado ao paciente que preenchesse a escala auto-aplicável sobre seu nível de ansiedade e foram mensurados os parâmetros clínicos. Finda a coleta inicial dos dados, realizou-se o procedimento terapêutico no paciente voluntário. A aplicação da técnica deu-se na enfermaria, no próprio leito do paciente, com o mesmo deitado e respeitando-lhe a privacidade e num intervalo de uma hora e meia e trinta minutos antes do horário de entrada no centro cirúrgico.
A calatonia é uma técnica de relaxamento composta de toques sutis em nove pontos do corpo, demorando-se de um a três minutos em cada ponto ${ }^{(16)}$. São considerados pontos: regiões ungueais de todos os artelhos (dedos dos pés), as plantas dos pés, os tornozelos, as convergências tendinosas do tríceps sural, das regiões posteriores das pernas (panturrilhas) e a região posterior do pescoço (nuca). O processo é realizado em silêncio, ocorrendo apenas o toque das mãos e dos dedos sem pressão ou fricção nos pontos referidos. Para a seqüência dos toques, é necessária que seja feita a identificação dos dedos das mãos do aluno e dos dedos dos pés do paciente, pois além da seqüência dos toques, deve-se respeitar a correspondência dos dedos (aluno-paciente), como mostra a Figura 1.

Os toques são feitos simultaneamente nos dois lados do corpo do paciente, todos com a mesma duração, com orientação caudal-cefálica, tocando-se, em forma de pinça, cada dedo do pé do paciente com o dedo da mão do aluno correspondente, como mostrado na Figura 2: $1^{\circ}$ ) Dedo 3 das mãos com dedo 3 dos pés; $2^{\circ}$ ) Dedo 2 das mãos com dedo 2 dos pés; $3^{\circ}$ ) Dedo 4 das mãos com dedo 4 dos pés; $4^{\circ}$ ) Dedo 5 das mãos com dedo 5 dos pés; $5^{\circ}$ ) Dedos 2, 3, 4 e 5 das mãos com dedo 1 dos pés; $\left.6^{\circ}\right)$ Após a aplicação nos dedos, os próximos pontos são tocados com as palmas das mãos, começando pelas plantas dos pés do paciente; $7^{\circ}$ ) Tornozelos; $8^{\circ}$ ) Panturrilhas; $9^{\circ}$ ) Finalizando na nuca, podendo ser utilizadas as palmas das mãos ou as pontas dos dedos. Para o grupo experimental (calatonia) utilizaram-se os pontos referidos; já para o grupo controle os pontos foram outros, como mostrados na Figura 3.

Salienta-se que, para ambos os grupos, a duração do toque em cada ponto foi de 1 minuto, isto é, a aplicação da técnica durou, aproximadamente, 10 minutos para cada indivíduo. O toque no grupo controle não obedeceu, a rigor, os pontos pré-definidos; contudo, foram tocados
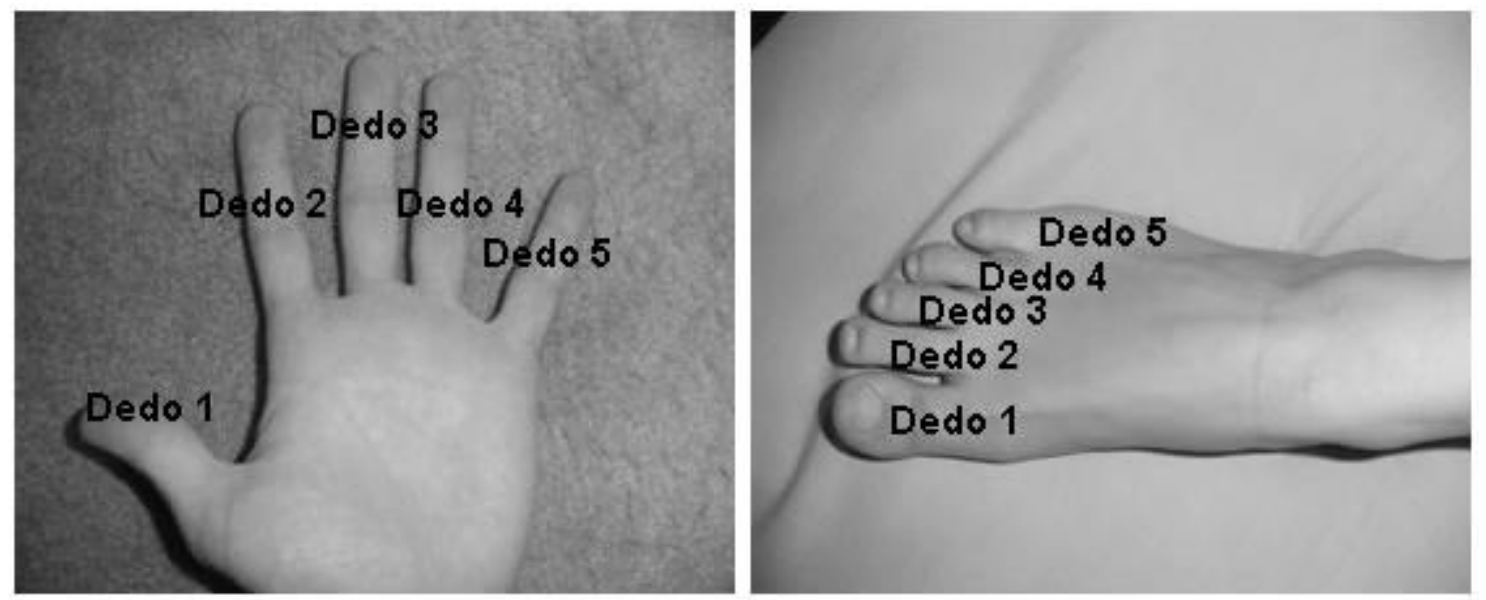

FIGURA 1 - Identificaçăo dos dedos da mão do aluno e dos pés do paciente 

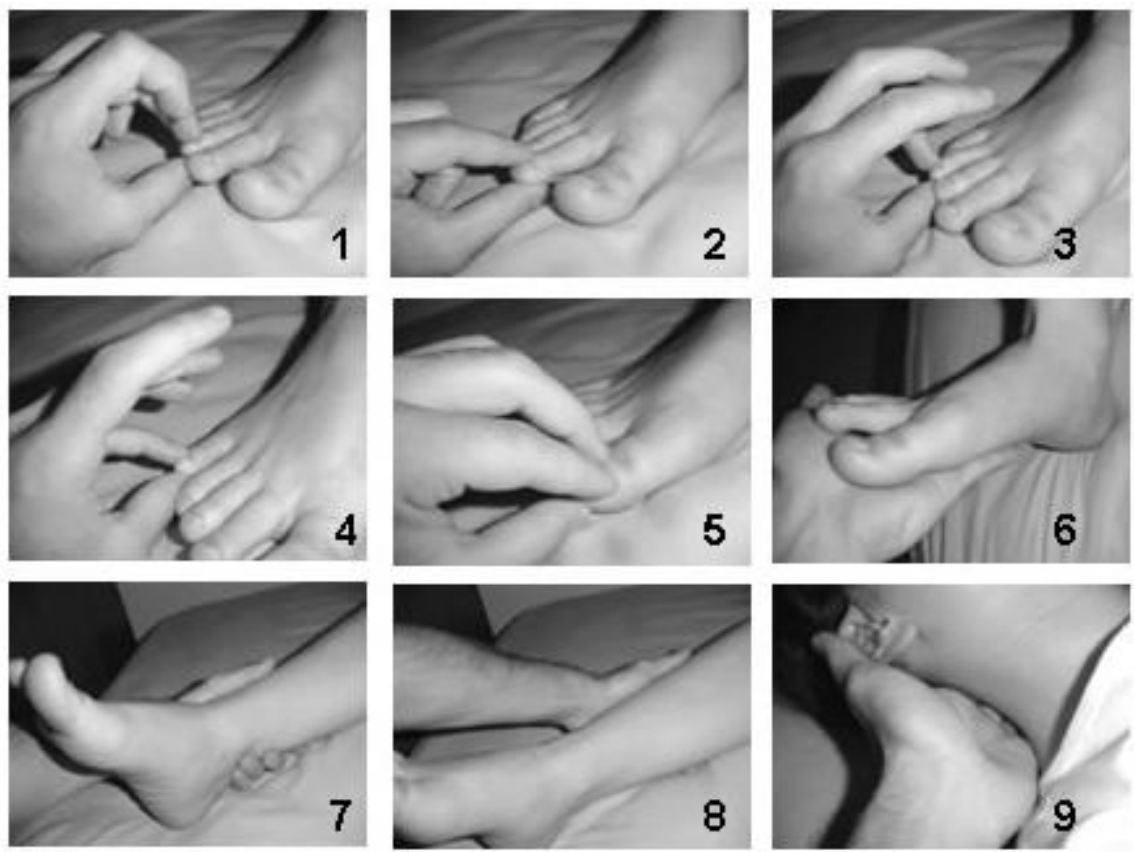

FIGURA 2 - Sequêencia dos toques: (1) dedos 3 das mãos com 3 dos pés (região ungueal), (2) dedos 2 das mâos com 2 dos pés (regiâo ungueal), (3) dedos 4 das mãos com 4 dos pés (região ungueal), (4) dedos 5 das mắos com 5 dos pés (região ungueal), (5) dedos $2,3,4 \mathrm{e} 5$ das máos com 5 dos pés (região ungueal), (6) mäos com solas dos pés, (7) mãos com tornozelos (face posterior) ( 8 ) măos com panturrilhas (face posterior), (9) dedos $2,3,4 \mathrm{e} 5$ das mấos com nuca.
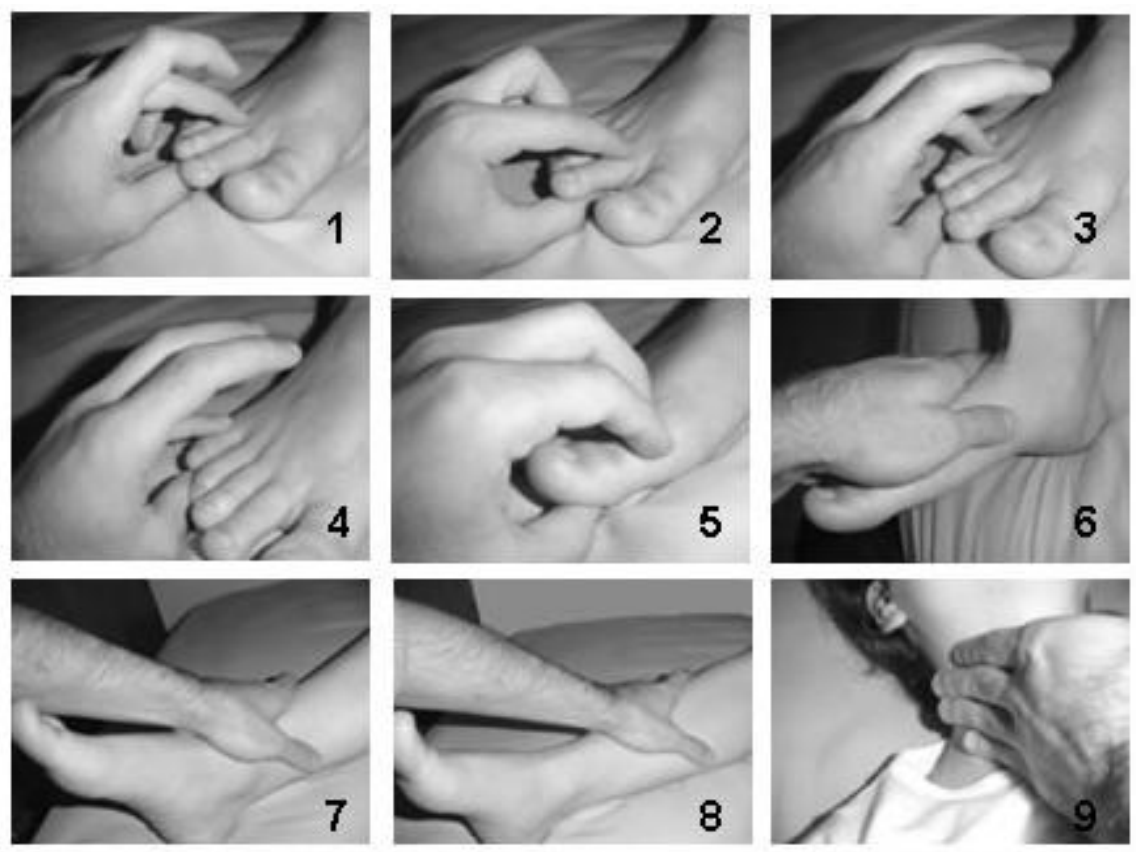

FIGURA 3 - Sequeência dos toques: (1) dedos 3 das mãos com 3 dos pés, (2) dedos 2 das mãos com 2 dos pés, (3) dedos 4 das mãos com 4 dos pés, (4) dedos 5 das mãos com 5 dos pés, (5) dedos $2,3,4$ e 5 das mâos com 5 dos pés, (6) mãos com dorsos dos pés, (7) mâos com tornozelos (face anterior), (8) măos com panturrilhas (face anterior), (9) dedos $2,3,4$ e 5 das mãos com nuca (faces laterais).

os mesmos sítios dérmicos mencionados no Termo de Consentimento Livre e Esclarecido. Após a aplicação da técnica, conversou-se com o paciente com o intuito de inquirir as alterações e/ou sensações percebidas durante a aplicação da técnica, bem como para esclarecer possíveis dúvidas e curiosidades. Finalizou-se a coleta com a mensuração dos parâmetros vitais.

Para o tratamento estatístico foram utilizados os testes Mann-Whitney, de Fischer e Qui-quadrado com significância e $\mathrm{p}<0,05$. 


\section{RESULTADOS}

No grupo calatonia, 9 indivíduos $(30 \%)$ encontraramse na faixa etária entre 18-25 anos; $5(16,67 \%)$ entre 2635 anos; $3(10 \%)$ entre 36-45 anos; 9 (30\%) entre 46-60 anos e $4(13,33 \%)$ na faixa etária superior a 60 anos. Com relação ao grupo controle, 5 indivíduos $(33,33 \%)$ na faixa etária entre 18-25 anos; 4 (26,67\%) entre 26-35 anos; 3 (20\%) entre 36-45 anos; $2(13,33 \%)$ entre 46-60 anos e 1 $(6,67 \%)$ na faixa etária superior a 60 anos.

Em relação ao gênero, no grupo calatonia, a amostra constituiu-se de 15 indivíduos (50\%) do sexo masculino e $15(50 \%)$ do sexo feminino. Já no grupo controle, a amostra constituiu-se de 8 indivíduos $(53,33 \%)$ do sexo masculino e 7 (46,67\%) do sexo feminino.

Referente à realização de cirurgia prévia, os dois grupos apresentam-se da mesma forma, isto é, no grupo calatonia, 16 indivíduos (53,3\%) haviam realizado algum tipo de cirurgia anterior, enquanto 14 (46,7\%) nunca haviam realizado algum tipo de cirurgia. No grupo controle, 11 indivíduos $(73,3 \%)$ haviam realizado algum tipo de cirurgia anterior e $4(26,7 \%)$ nunca haviam realizado algum tipo de cirurgia. Do total dos indivíduos que já haviam se submetido a algum tipo de cirurgia, apenas $1(3,7 \%)$ apresentou algum tipo de intercorrência intra ou pós operatória.

A ansiedade apresentou-se na seguinte distribuição:

Tabela 1 - Distribuição da ansiedade nos grupos calatonia e controle. São Paulo, 2005.

\begin{tabular}{lccc}
\hline \multirow{2}{*}{ Ansiedade } & \multicolumn{3}{c}{ Grupos } \\
\cline { 2 - 4 } & $\begin{array}{c}\text { Calatonia } \\
(\mathbf{n}=\mathbf{3 0})\end{array}$ & $\begin{array}{c}\text { Controle } \\
(\mathbf{n}=\mathbf{1 5})\end{array}$ & $\begin{array}{c}\text { Total } \\
\mathbf{( n = 4 5 )}\end{array}$ \\
\hline Baixa & $18(60 \%)$ & $10(66,7 \%)$ & $28(62,2 \%)$ \\
Média & $12(40 \%)$ & $5(33,3 \%)$ & $17(37,8 \%)$ \\
Alta & $0(0,0 \%)$ & $0(0,0 \%)$ & $0(0,0 \%)$ \\
\hline
\end{tabular}

Dos 30 indivíduos do grupo calatonia, 18 (60\%) apresentaram baixa ansiedade e 12 (40\%) média ansiedade. O grupo controle obteve uma amostra com 10 indivíduos $(66,7 \%)$ classificados em baixa ansiedade e 5 (33,3\%) classificadas em média ansiedade. No total da amostra, 28 indivíduos $(62,2 \%)$ apresentaram baixa ansiedade e 17 $(37,8 \%)$ apresentaram média ansiedade. Para todas as variáveis apresentadas, os grupos mostraram-se homogêneos estatisticamente.

Os dados da Tabela 2 mostram como os parâmetros vitais do grupo controle e experimental se expressaram antes e após a aplicação da técnica de relaxamento. A análise dos dados referentes aos sinais vitais foi realizada da seguinte forma: pareamento dos sinais vitais referentes a cada grupo, utilizando-se a média dos valores no pré e no pós a aplicação da técnica de relaxamento, mostrado na Tabela 2; comparação entre os respectivos valores para os respectivos grupos mostrada na Tabela 3.

Tabela 2 - Distribuição dos parâmetros vitais nos grupos calatonia e controle, mensurados antes e após a aplicação da técnica de relaxamento. São Paulo, 2005.

\begin{tabular}{|c|c|c|c|c|c|}
\hline \multirow{3}{*}{$\begin{array}{l}\text { Sinais } \\
\text { Vitais }\end{array}$} & \multicolumn{4}{|c|}{ Grupos } & \multirow{3}{*}{ Pares } \\
\hline & \multicolumn{2}{|c|}{$\begin{array}{c}\text { Calatonia } \\
(n=30)\end{array}$} & \multicolumn{2}{|c|}{ Controle $(n=15)$} & \\
\hline & Média & $\begin{array}{l}\text { Desvio } \\
\text { Padrão }\end{array}$ & Média & $\begin{array}{l}\text { Desvio } \\
\text { Padrão }\end{array}$ & \\
\hline $\begin{array}{l}\text { Freq. Pulso } \\
\text { PRÉ }\end{array}$ & 68.70 & 10.986 & 68.67 & 10.376 & \\
\hline $\begin{array}{l}\text { Freq. Pulso } \\
\text { PÓS }\end{array}$ & 65.30 & 7.983 & 66.20 & 11.143 & 1 \\
\hline $\begin{array}{l}\text { PA Sistólica } \\
\text { PRÉ }\end{array}$ & 109.50 & 16.461 & 116.80 & 18.135 & \\
\hline $\begin{array}{l}\text { PA Sistólica } \\
\text { PÓS }\end{array}$ & 108.63 & 17.582 & 116.33 & 12.111 & 2 \\
\hline $\begin{array}{l}\text { PA } \\
\text { Diastólica }\end{array}$ & 74.67 & 11.442 & 77.33 & 13.372 & \\
\hline $\begin{array}{l}\text { PRE } \\
\text { PA }\end{array}$ & & & & & 3 \\
\hline $\begin{array}{l}\text { Diastólica } \\
\text { PÓS }\end{array}$ & 74.37 & 10.159 & 73.80 & 11.906 & \\
\hline $\begin{array}{l}\text { Freq. Resp. } \\
\text { PRE }\end{array}$ & 19.00 & 3.705 & 20.00 & 4.276 & \\
\hline $\begin{array}{l}\text { Freq. Resp. } \\
\text { PÓS }\end{array}$ & 18.60 & 4.116 & 20.67 & 4.047 & 4 \\
\hline $\begin{array}{l}\text { Temperatura } \\
\text { PRÉ }\end{array}$ & 35,90 & 0,562 & 36,15 & 0,463 & \\
\hline $\begin{array}{l}\text { Temperatura } \\
\text { PÓS }\end{array}$ & 35,97 & 0,486 & 36,14 & 0,500 & 5 \\
\hline
\end{tabular}

Tabela 3 - Distribuição dos grupos calatonia e controle, segundo o pareamento dos sinais vitais. São Paulo, 2005.

\begin{tabular}{|c|c|c|c|}
\hline Grupos & Pares & Sinais Vitais & $\begin{array}{l}\text { Significância } \\
\text { (p) }\end{array}$ \\
\hline \multirow{5}{*}{ Controle } & Par 1 & Pulso-PRÉ \& Pulso-PÓS & 0,059 \\
\hline & Par 2 & PAmax-PRÉ \& PAmax-PÓS & 0,899 \\
\hline & Par 3 & PAmin-PRÉ \& PAmax-PÓS & 0,223 \\
\hline & Par 4 & Resp-PRÉ Resp-PÓs & 0,417 \\
\hline & Par 5 & Temp-PRÉ \& Temp-PÓs & 0,934 \\
\hline \multirow{5}{*}{ Calatonia } & Par 1 & Pulso-PRÉ \& Pulso-PÓS & 0,015 \\
\hline & Par 2 & PAmax-PRÉ \& PAmax-PÓS & 0,463 \\
\hline & Par 3 & PAmin-PRÉ \& PAmax-PÓS & 0,853 \\
\hline & Par 4 & Resp-PRÉ \& Resp-PÓS & 0,604 \\
\hline & Par 5 & Temp-PRÉ \& Temp-PÓs & 0,368 \\
\hline
\end{tabular}

Dos indivíduos pertencentes ao grupo calatonia, antes da aplicação da técnica, apresentaram média de freqüência 
de pulso de 68,70 bpm, pressão arterial sistólica de 109,50 $\mathrm{mmHg}$, pressão arterial diastólica de 74,67 $\mathrm{mmHg}$, freqüência respiratória de 19,00 $\mathrm{rpm}$ e temperatura de $35,90{ }^{\circ} \mathrm{C}$. O grupo controle, antes da aplicação da técnica, apresentou média de freqüência de pulso de 68,67 bpm, pressão arterial sistólica de 116,80 $\mathrm{mmHg}$, pressão arterial diastólica de $77,33 \mathrm{mmHg}$, freqüência respiratória de 20,00 rpm e temperatura de $36,15^{\circ} \mathrm{C}$. Para os valores das médias após a aplicação da técnica, o grupo calatonia obteve freqüência de pulso de $65,30 \mathrm{bpm}$, pressão arterial sistólica de 108,63 mmHg, pressão arterial diastólica de $74,37 \mathrm{mmHg}$, freqüência respiratória de 18,60 rpm e temperatura de $35,97^{\circ} \mathrm{C}$. Já para o grupo controle as médias foram freqüência de pulso de $66,20 \mathrm{bpm}$, pressão arterial sistólica de $116,33 \mathrm{mmHg}$, pressão arterial diastólica de $73,80 \mathrm{mmHg}$, freqüência respiratória de 20,67 rpm e temperatura de $36,14{ }^{\circ} \mathrm{C}$.

Com exceção da temperatura referente ao grupo calatonia e a freqüência respiratória do grupo controle, os parâmetros clínicos obtidos após a aplicação da técnica de relaxamento foram consideravelmente menores, tanto no grupo controle como do grupo calatonia, embora não significantes estatisticamente.

Destaca-se, nestes dados, a freqüência de pulso que se apresentou estatisticamente significante $(p=0,015)$.

\section{DISCUSSÃO}

Acredita-se que a atuação direta do Sistema Nervoso Autônomo no coração propicia rápidas modificações no dromotropismo do músculo cardíaco, refletindo rapidamente em alterações mensuráveis na freqüência de pulso e, conseqüentemente, na pressão arterial e na freqüência respiratória, embora esta correspondência não tenha sido visível numericamente neste estudo. Os resultados obtidos com a análise dos sinais vitais permitem afirmar que a técnica exerceu efeito positivo sobre o estado de ansiedade, isto é, a aplicação da técnica no paciente pré-cirúrgico contribuiu para a redução da sua ansiedade. Contudo, como explicar a alteração significante apenas nos valores referentes à freqüência de pulso, sabendo que pressão arterial e freqüência respiratória estão diretamente ligadas à mesma? Algumas inferências foram feitas durante a análise dos dados, isto é, a pressão arterial, apesar de mostrar redução após a aplicação da técnica nos dois grupos, não foi significante estatisticamente. Isto talvez devido ao pequeno período entre o término da técnica e a mensuração dos sinais vitais, no qual eram respondidas algumas dúvidas e relatadas algumas impressões referentes à calatonia; a freqüência respiratória apresentou diminuição somente no grupo calatonia, o que talvez corresponda à diminuição significante da freqüência de pulso. Observou-se também ligeiro aumento da temperatura corporal pós-técnica, o que leva a associar ao relaxamento da musculatura dos vasos periféricos, aumentando o fluxo sangüíneo e elevando a temperatura; a aplicação da técnica em 10 minutos provavelmente produziu efeitos menos intensos do que se a mesma tivesse sido realizada em 30 minutos. O tempo para aplicação foi suficiente para a grande maioria dos indivíduos desligarem-se da suas preocupações ou, ao menos, encará-las de forma mais confiante. Embora alguns pacientes tenham relatado informalmente ser esse tempo (10 minutos) suficiente apenas para o início do processo de relaxamento. Sendo assim, a expansão do tempo de aplicação, talvez possibilitasse outros resultados Como solução para este problema relacionado ao tempo de aplicação, acredita-se que 3 aplicações contínuas, isto é, 3 aplicações de 10 minutos cada, intercaladas e respeitando a necessidade de cada um, traria resultados significantes no relaxamento proposto.

Sugere-se associar instrumentos que pontuem sensações de bem estar associadas ao alívio da ansiedade, uma vez que muitos pacientes relataram além do relaxamento muscular, sensação de calor e formigamento nos membros inferiores. São necessárias, ainda, ferramentas que identifiquem suas estratégias de enfrentamentos, pois é provável que as oscilações dos parâmetros clínicos avaliados não tenham sido tão acentuadas devido aos mecanismos de coping desenvolvidos pelos indivíduos.

\section{CONCLUSÃO}

No que se refere à ansiedade relacionada ao grupo calatonia e ao grupo controle, os indivíduos apresentaram em sua maioria baixa ansiedade. Os parâmetros clínicos obtidos após a aplicação da técnica de relaxamento foram consideravelmente menores, tanto do grupo controle como do grupo calatonia, embora não significantes estatisticamente, com exceção da freqüência de pulso $(\mathrm{p}=0,015)$.

\section{REFERÊNCIAS}

1. Nagai SC. Toque terapêutico. Revista SOBECC. 2002; 7(4): 9-12.

2. Gala MF, Telles SCR, Silva MJP. Ocorrência e significado do toque entre profissionais de enfermagem e pacientes de uma UTI e Unidade Semi-Intensiva cirúrgica. Rev Esc Enfermagem USP. 2003; 37(1): 52-61.

3. Albrecht K. A fisiologia do estresse. In: Albrecht K. O gerente e o estresse: faca o estresse trabalhar para você. $2 \mathrm{a}$ ed. Rio de Janeiro: Jorge Zahar; 1990. p.51-80.

4. Faintuch J. Observação clínica pré-operatória. In: Faintuch J, Raia AA. Manual de pré e pós-operatório. São Paulo: Manole; 1978. cap. 1. p.3-13.

5. Hoffer JL. Anestesia. In: Meeker MH, Rothrock JC. Alexander cuidados de enfermagem ao paciente cirúrgico. 10a ed. Rio de Janeiro: Guanabara Koogan; 1997. cap. 6. p.134-68. 
6. Spielberger CD, Gorsuch RL, Lushene RE. Inventário de ansiedade traço-estado-idate. Rio de Janeiro: Cepa; 1979.

7. Chaves EC. Stress e trabalho do enfermeiro: a influência de características individuais no ajustamento e tolerância ao turno noturno [tese]. São Paulo: Instituto de Psicologia da Universidade de São Paulo; 1994.

8. DeLucia R, Planeta CS. Ansiolíticos. In: Valle LBS, Oliveira Filho RM, DeLucia R, coordenadores. Farmacologia integrada. Rio de Janeiro: Atheneu; 1991. cap. 7. p.87-91.

9. Aldrete JA, Kroulik D. A postanesthetic recovery score. Anesth Analg. 1970; 49(6):924-34.

10. Posso MBS. Avaliação das condições dos pacientes na sala de recuperação pós-anestésica. Rev Esc Enfermagem USP. 1975; 9(3):9-23.
11. Beland IL, Passos JY. Defesas e respostas do corpo contra a agressão. In: Beland IL, Passos JY. Enfermagem clínica: aspectos fisiopatológicos e psicossociais. São Paulo: Epu; 1978. cap. 7. p.319-79.

12. Bevilacqua RG. Alterações endócrinas e metabólicas no trauma. In: Allgower M, Bevilacqua RG. Manual de cirurgia. 2a ed. São Paulo: EPU; 1981. cap.2. p.21-33.

13. Castanos CC. Índices de recuperação. Rev Bras Anestesiol. 1982; 32(6): 441-2.

14. Schneider M. Meeting the criterio for discharge. Curr Rev Recov Room Nurses. 1982; 4(6): 41-8.

15. Griffin DN. A tool to develop standards of quality care in PACUs. J Post Anesth Nurs. 1989; 4(2):99-102.

16. Sandor P. Técnicas de relaxamento.São Paulo: Vetor; 1974. 